\title{
Development of the pneumatic system with an annular aspiration channel for vibrating and rotation grain separator
}

\author{
Vasiliy L. Andreev ${ }^{1, *}$, Vladimir V. Shilin ${ }^{2}$, Valeriy V. Ilichev ${ }^{1}$, and Tatjana S. Holodenina ${ }^{1}$ \\ ${ }^{1}$ Nizhniy Novgorod state engineering-economic university, 22A, Oktyabrskaya street, Knyaginino, \\ 606340, Russia \\ ${ }^{2}$ Vyatka state agrarian-technological university, 133, Oktyabrsky avenue, Kirov, 610017, Russia
}

\begin{abstract}
The pneumatic system with a vertical annular aspiration channel for vibration-centered grain separators been developed. The rational values of the disk grain distributor are the length of the sectors $0.15 \mathrm{~m}$, the angle of inclination of the sectors $60^{\circ}$. The rotation speed of the distributor in the seed mode (grain supply $5 \ldots 15 \mathrm{t} / \mathrm{h}$ ) should be $100 \ldots 200 \mathrm{~min}^{-1}$ and in the food mode (grain supply $15 \ldots 25 \mathrm{t} / \mathrm{h}$ ) must be $100 \ldots 150 \mathrm{~min}^{-1}$. The effect of the release of light impurities in the developed aspiration system decreases from $45 \%$ to $20 \%$ with an increase in the supply of grain G from 5 to $25 \mathrm{t} / \mathrm{h}$, herewith at the same time the loss of a full-fledged grain to waste decreases from $0.6 \ldots 0.8 \%$ to $0.3 \ldots 0.4 \%$, while the effect of the release of light impurities in the aspiration system of the mass-produced vibration centrifugal grain separator decreases from $11 \%$ to $4 \%$ with the same losses of grain to waste.
\end{abstract}

\section{Introduction}

Larger agricultural enterprises have higher grain yields [1]. Therefore the use of highly efficient and capacity machines for seed and grain cleaning is important.

The most widespread in agro-industrial enterprises of the Russian Federation are the air and sieve grain cleaning machines [2]. To separate the source material into fractions these machines use a gravity field, so they have a less specific productivity of the sieves surface.

Centrifugal separators have higher values of specific productivity in which the exit of particles through the separating surface of the sieves occurs under the action of inertial forces far exceeding gravity [3].

Currently a large number of the vibration centrifugal grain separators of various brands are being mass-produced [4-7 and others]. For example the A1-BCS-100 vibrating grain separator [8] is designed to isolate impurities from grain material that differ in thickness, width and aerodynamic properties (figure 1).

This separator has four identical blocks. Each block consists of a housing 6, an annular aspiration channel 3 , a rotating rotor with a sieve drum of three separate cylindrical sieves $5,9,10$ and sieves cleaning devices 7,13 .

\footnotetext{
*Corresponding author: andreev.vas@mail.ru
} 


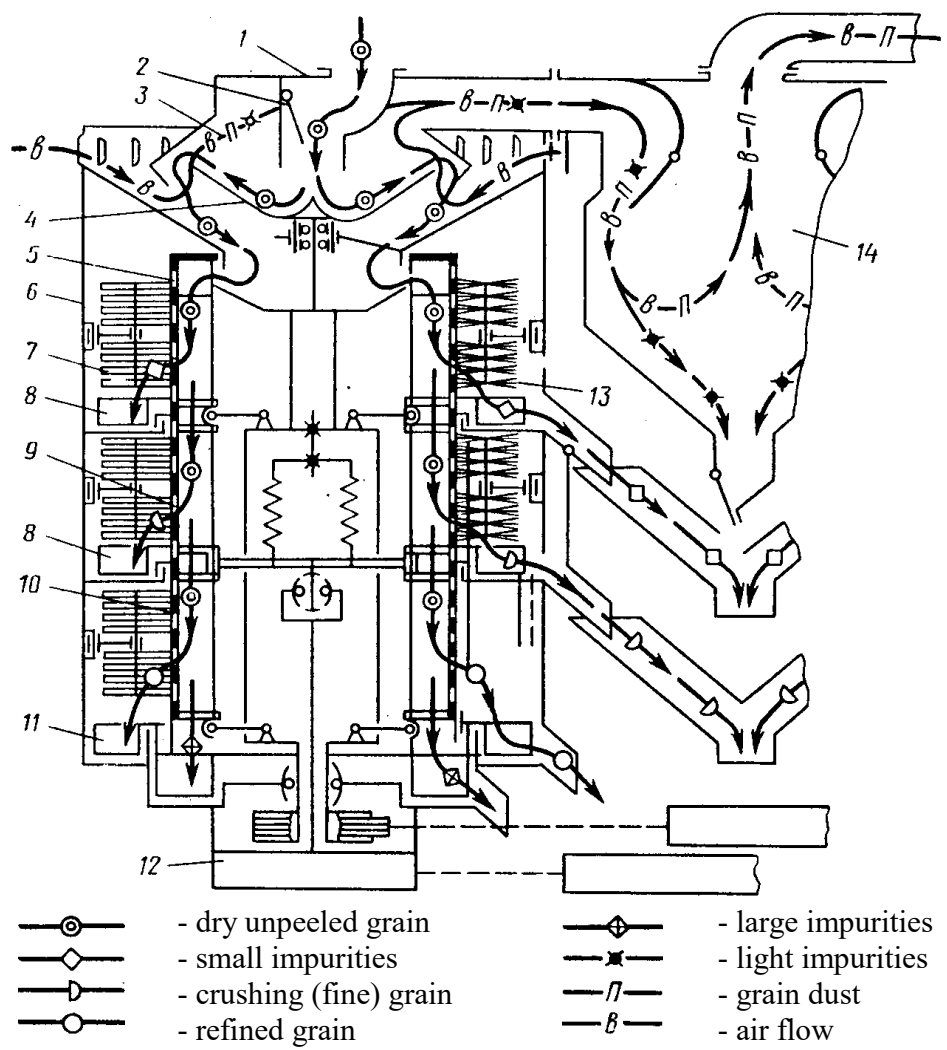

Fig. 1. Technological scheme of one block of separator unit A1-BCS-100: 1 - dispenser; 2 - valve; 3 - aspiration channel; 4 - disc feeder; 5, 9, 10 - sieves; 6 - housing; 7 - rubber cleaner; 8,11 blades; 12 - vibrator; 13 - brush cleaner; 14 - sedimentary chamber

This separator has four identical blocks. Each block consists of a housing 6, an annular aspiration channel 3 , a rotating rotor with a sieve drum of three separate cylindrical sieves $5,9,10$ and sieves cleaning devices 7,13 .

The grain material through the dispenser 1 enters the disc feeder 4, which evenly feeds grain into the annular aspiration channel 3. Light impurities are released in the channel 3 and carried out with the air flow into the sedimentary chamber 14, where the bulk of them settles and is removed. At the same time the internal cavity of the sieve drum is aspirated. The remaining light impurities are released in the cyclone which has a high cleaning efficiency, so that the concentration of grain dust in the air of the working area does not exceed the permissible norms.

Next the grain material flows by gravity to a rotating distribution device that moves the material to the upper sieve 5 that releases small impurities. Due to the rotational and oscillatory motion of the sieve drum, the grain mixture moves in a uniform layer along the sieves from top to bottom. Small impurities pass through the sieve apertures 5, while the grain material is moved to grading sieve 9. The puny and part of the crushed grain passes through the holes of the sieve 9, the cleaned material with large impurities moves to the sieve 10, where the clean grain passes through the holes and is removed from the machine, and large impurities go down along the lower sieve 10 .

However this separator has some disadvantages.

The grain material in the annular aspiration channel 3 is processed once at an increased speed, but for a short time, since the channel 3 formed by the disc feeder 4 and the feeder body has a short length, as a result of which light impurities not have time to separate from 
the cleaned material.

Also the channel has different hydraulic resistance along its length due to the location of the air intake, which is located on the side wall of the housing 6 , which causes a large unevenness of the air velocity in the channel.

Too the device for feeding grain material into the annular aspiration channel has not been sufficiently studied.

\section{Materials and methods}

The pneumatic system with a vertical annular aspiration channel for the vibration centrifugal grain separator has been developed and its experimental sample has been manufactured (figure 2).
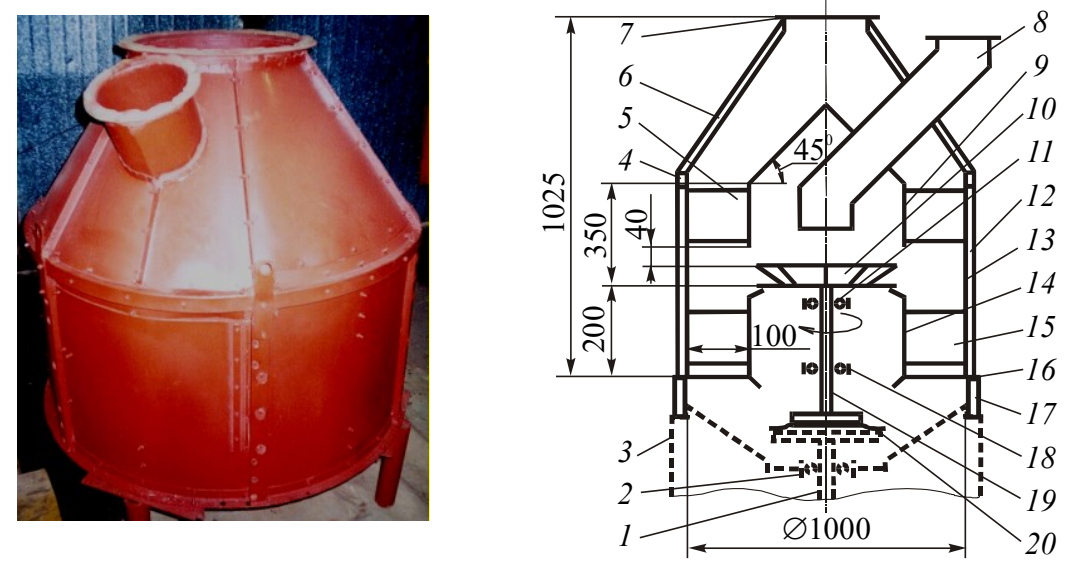

Fig. 2. Pneumatic system with a vertical annular aspiration channel and with a disk grain distributor with inclined sectors: $\mathrm{a}$ - general view; $\mathrm{b}$ - schema; 1 - the shaft of the sieve separator; $2,11,18$ bearing support; 3 - the housing of the sieve separator; 4 - the upper ring of the housing of the pneumatic system; 5 - the upper spacer plate; 6 - the housing of the pneumatic system; 7 - the mounting flange of the duct for removing exhaust air; 8 - the grain loading pipeline; 9,14 - the inner wall of the aspiration channel; 10 - the disc grain distributor; 12 - the lower housing rack of pneumatic system; 13 - the outer wall of the aspiration channel; 15 - the lower spacer plate; 16 - the lower ring of the pneumatic system housing; 17 - the rack of the sieve separator housing; 19 - the shaft of the grain distributor disk; 20 - coupling

Through grain loading pipeline 8 the grain mixture is fed to the rotating disk grain distributor 10 with replaceable sectors. Under the action of centrifugal forces the cleaned material moves first along the horizontal surface and then along the inclined sectors of the distributor 10 enters the separation zone of the vertical annular aspiration channel formed by the outer 13 and inner 9, 14 walls. Here the cleaned material is blown by the air flow created by the fan, which is not shown in the figure. The light impurities trapped in the aspiration channel 8 are removed together with the exhaust air through an air duct connected to the flange 7. Spacer plates 5, 15 of the same length provide the same depth of the vertical annular aspiration channel along its length and height in the separation zone. Exhaust air intake is carried out from the upper part of the pneumatic system. The outer diameter of the vertical annular channel had a value of $1.0 \mathrm{~m}$ and its depth was $0.1 \mathrm{~m}$.

Rotation frequency 10 grain disk distributor was values $n=100 \ldots 250 \mathrm{~min}^{-1}$, but changed using an electric motor and a DC source. The angle of inclination of the sectors had meanings $\alpha=0 \ldots 60^{\circ}$, and their length was $l=0 \ldots 0,20 \mathrm{~m}$ (fig. 3). 

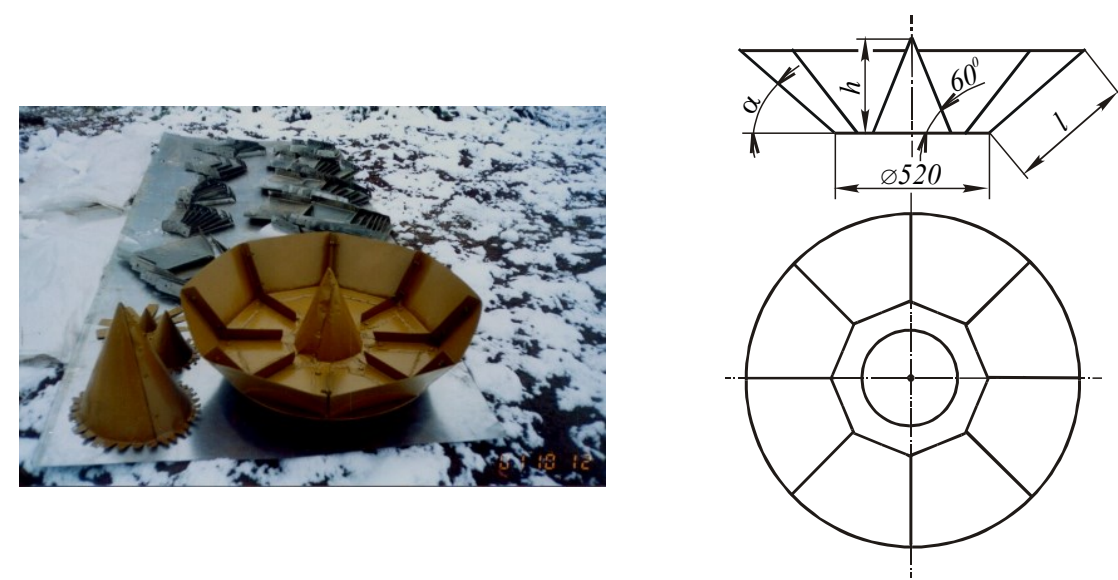

Fig. 3. The rotating disk grain distributor with replaceable sectors

\section{Results and Discussion}

To determine the rational parameters of the disk distributor when it is functioning in the aspiration system, a two-level central composite rotatable plane $2^{4-1}$ of the first-order experiment for four factors [9] is implemented. After the implementation of the experiment plan, regression models were obtained, but which do not adequately describe the real processes (used F-criterion of Fisher at the probability $p=0,95$ ).

Therefore further implemented is a three-level plan for the second-order BoxBenkin experiment for four factors [9]. As the main culture, the barley seeds of the Abava variety with a thickness of more than $2.4 \mathrm{~mm}$ in an amount of $95 \%$ were used, and the impurity was small rye with a thickness of less than $2.0 \mathrm{~mm}$ in an amount of $5 \%$. Factors and levels of their variation are shown in table 1.

Table 1. Factors and levels of their variation when implementing an experiment of Box-Benkin's plan of the second order for four factors

\begin{tabular}{|c|l|c|c|c|c|c|c|}
\hline \multirow{2}{*}{$\begin{array}{c}\text { Coded value } \\
\text { of factors }\end{array}$} & \multirow{2}{*}{$\begin{array}{c}\text { The name of the factors, their } \\
\text { designation and unit of } \\
\text { measurement }\end{array}$} & \multicolumn{6}{|c|}{ Factor levels } \\
\cline { 3 - 9 } & & \multicolumn{4}{|c|}{ Seed mode } & \multicolumn{4}{|c|}{ Food mode } \\
\cline { 3 - 9 } & & -1 & 0 & 1 & -1 & 0 & 1 \\
\hline$x_{1}$ & Grain G supply, $\mathrm{t} / \mathrm{h}$ & 5 & 10 & 15 & 15 & 20 & 25 \\
\hline$x_{2}$ & $\begin{array}{l}\text { Frequency } n \text { of rotation of the } \\
\text { distributor, } \text { min }^{-1}\end{array}$ & 100 & 175 & 250 & 100 & 175 & 250 \\
\hline$x_{3}$ & Lenght $l$ of sector, $\mathrm{m}$ & 0 & 0,075 & 0,150 & 0 & 0,075 & 0,150 \\
\hline$x_{4}$ & Angle $\alpha$ of sector, degree & 0 & 30 & 60 & 0 & 30 & 60 \\
\hline
\end{tabular}

The optimization criteria adopted effects of selection of impurities from the grain mixture on the seed $E_{S E E D}$ and food $E_{F O O D}(\%)$ modes, as well as losses $a_{S E E D}, a_{F O O D}(\%)$ of full grain in waste.

After the implementation of the experiment's plan, regression models were obtained, which adequately describe the actual processes (used F-criterion of Fisher at the probability $p=0,95)$ :

$$
\begin{aligned}
E_{\text {SEED }} & =23,43-5,51 \cdot x_{1}+3,92 \cdot x_{3}+4,52 \cdot x_{4}+1,38 \cdot x_{1}^{2}+0,415 \cdot x_{1} x_{2}-0,79 \cdot x_{1} x_{4}- \\
& -0,05 \cdot x_{2}^{2}+0,79 \cdot x_{2} x_{3}+0,44 \cdot x_{2} x_{4}+0,92 \cdot x_{3}^{2}+4,08 \cdot x_{3} x_{4}+1,88 \cdot x_{4}^{2}
\end{aligned}
$$

$a_{\text {SEED }}=0,137-0,040 \cdot x_{1}+0,104 \cdot x_{2}+0,173 \cdot x_{3}+0,160 \cdot x_{4}-0,011 \cdot x_{1}^{2}-0,020 \cdot x_{1} x_{2}-0,040 \cdot x_{1} x_{3}$

$-0,024 \cdot x_{1} x_{4}+0,017 \cdot x_{2}^{2}+0,088 \cdot x_{2} x_{3}+0,093 \cdot x_{2} x_{4}+0,054 \cdot x_{3}^{2}+0,230 \cdot x_{3} x_{4}+0,330 \cdot x_{4}{ }^{2}$; 


$$
\begin{gathered}
E_{F O O D}=15,76-2,17 \cdot x_{1}-0,21 \cdot x_{2}+2,80 \cdot x_{3}+3,45 \cdot x_{4}+0,40 \cdot x_{1}^{2}-0,31 \cdot x_{1} x_{3}-1,10 \cdot x_{1} x_{4}+ \\
\quad+0,06 \cdot x_{2}^{2}+0,25 \cdot x_{2} x_{3}+1,07 \cdot x_{2} x_{4}-0,84 \cdot x_{3}^{2}+2,82 \cdot x_{3} x_{4}+0,18 \cdot x_{4}^{2}
\end{gathered}
$$

Analysis of equations (1)...(4) showed that as on seed mode with changes in the pneumatic bandwidth $G$ from 5 to $15 \mathrm{t} / \mathrm{h}$, and on food regime when changes in the capacity of the pneumatic system $G$ from 15 to $25 \mathrm{t} / \mathrm{h}$ rational values are the length of the sectors $l=0,15 \mathrm{M}\left(x_{3}=1\right)$ and angle of inclination of sectors $\alpha=60^{\circ}\left(x_{4}=1\right)$, but the frequency of rotation of the grain disk distributor on seed mode (fig. 4, a) should be $n=100 \ldots 200$ $\min ^{-1}\left(x_{2}=-1 \ldots 0,33\right)$, but on food mode (fig. 4, b) should be $n=100 \ldots 150 \min ^{-1}\left(x_{2}=-\right.$ $1 \ldots$

$-0,33)$. Permissible losses of the main grain in waste with light impurities on seed mode $\left[a_{S E E D}\right]=1,0 \%$ and food mode $\left[a_{F O O D}\right]=0,5 \%$.
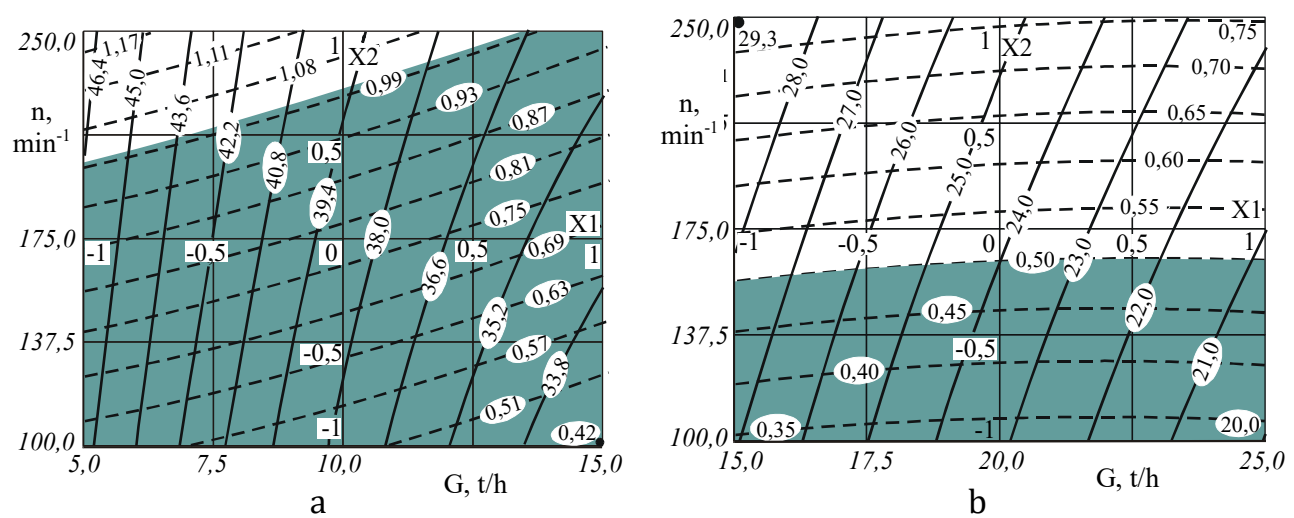

Fig. 4. The dependences of the effects of impurities and losses of full grain into waste during the functioning of the aspiration system from the supply $G$ of grain and the frequency $n$ of rotation of the distributor at the values of factors $x_{3}=1$ (the lenght of sectors $l=0,15$ м) и $x_{4}=1$ (The angle of inclination of sectors $\left.\alpha=60^{\circ}\right)$; $\mathrm{a}$ - on seed mode; $\mathrm{b}$ - on food mode; —— - effects $E_{\text {SEED }}$ and $E_{F O O D}$ of selection of impurities (\%); - - - - losses $a_{S E E D}$ and $a_{F O O D}$ basic grain in waste (\%); - areas of permissible grain loss $\left[a_{S E E D}\right]=1,0 \%$ и $\left[a_{F O O D}\right]=0,5 \%$

The studies of the developed pneumatic system were carried out in comparison with the pneumatic system installed in the serial-produced vibration centrifugal separator MZP-25, having a bandwidth of $25 \mathrm{t} / \mathrm{h}$. To estimate the effectiveness of impurities is passed through both pneumatic systems, $500 \mathrm{~kg}$ of prepared grain mixture (95\% - the Abava barley seeds variety with a thickness of more than $2.4 \mathrm{~mm}$ and $5 \%$ - the small winter rye with a thickness of less than $2.0 \mathrm{~mm}$ ). The air flow rate in the pneumatic channels during experiments was not changed and installed at the beginning of the removal of the full grain into waste at the lowest seeds of the grain mixture, taking into account the allowable losses of the main grain. All highlighted light fraction, including a small number of barley grains and the main part of winter rye grains, was shot in the tissue filter. The separation of the mixture was performed on solids with rectangular openings $2.2 \times 20 \mathrm{~mm}$. The experiments were carried out in three-time repetition.

The dependence of the effect $E$ of removing of impurities in the serial-produced pneumatic system and in the developed pneumatic system with an annular aspiration 
channel from the supply $G$ of grain with the same losses $a$ of grain in waste shows at fig. 5.

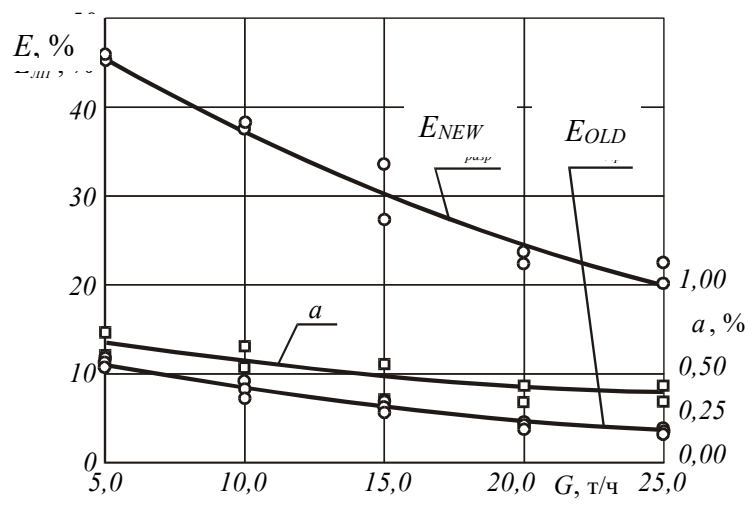

Fig. 5. The dependences of the effects of emission of lung impurities in the developed pneumatic system $E_{N E W}$ (length of the sectors of the distributor $l=0,15 \mathrm{~m}$, the angle of their tilt $\alpha=60^{\circ}$, rotation frequency $n=115 \mathrm{~min}^{-1}$ ) and serial produced pneumatic system $E O L D$ from the supply of $G$ grain with the same losses $a$ and the main grain in waste

With an increase in the supply $G$ of grain in aspiration systems from 5 to $25 \mathrm{t} / \mathrm{h}$ and simultaneously decreasing losses $a$ of a full grain in waste from $0,6 \ldots 0,8 \%$ to $0,3 \ldots 0,4 \%$ effect $E_{N E W}$ of election of light impurities in the developed pneumatic system decreases from $45 \%$ to $20 \%$, while the effect $E_{O L D}$ of the separation of light impurities in the serial produced pneumatic system is reduced from $11 \%$ to $4 \%$.

\section{Conclusion}

The developed pneumatic system with a vertical ring aspiration channel and a discdistributor of grain with inclined sectors more efficiently releases impurities from seed and food grain compared with the pneumatic system of mass-produced vibration centrifugal grain separators MZP-25 with the same losses of the main grain in waste.

\section{References}

1. S. Petrova, D. Zjukin, Assessment of factors for the increase in the productivity of grain crops

2. V.I. Orobinsky, A.M. Gievsky, V.A. Gulevsky, Prospects for improving the technology of post-harvest grain and seed processing and technical means to implement it

3. N.E. Avdeev, Centrifugal separators for grain, Moscow, Kolos, 152 (1975)

4. Vibration separators A1-BCS-100, https:// sinref.ru/

5. Vibration separators R8-BC2S and R8-UCS-200, https:// mukomoll.ru/

6. Grain centrifugal separators BCS-25, BCS-50, BCS-100, https:// melagrosnab.ru/

7. Centrifugal grain vibrating separator B1-VCS Belogorie, https://nizhniynovgorod.selhoztehnika.net/

8. B.V. Lebedev, Industrial seed processing and storage, Moscow, Agropromizdat, 255 (1991) 
9. S.V. Melnikov, V.R. Aljoshkin, P.M. Roshchin, Planning an experiment in agricultural process research, Leningrad, Kolos, 168 (1980) 